\title{
Comparison of diagnostic value of multi-slice spiral CT and MRI for different pathological stages of prostate cancer
}

\author{
YANBIN SUI ${ }^{*}$, JIAN LI $^{*}$, ZHENXING ZOU* ${ }^{*}$ YUNXIA SHI* and CUIJUAN HAO \\ Department of Medical Image, The Affiliated Yantai Yuhuangding Hospital of Qingdao University, \\ Yantai, Shandong 264000, P.R. China
}

Received November 21, 2018; Accepted April 3, 2019

DOI: $10.3892 / \mathrm{ol} .2019 .10272$

\begin{abstract}
Diagnostic value of magnetic resonance imaging (MRI) and multi-slice spiral CT (MSCT) for different pathological stages of prostate cancer was compared. A total of 112 patients with prostate cancer who underwent surgical pathology in The Affiliated Yantai Yuhuangding Hospital of Qingdao University from February 2014 to January 2016 were enrolled as prostate cancer group, and another 100 patients who received physical health examinations during the same period as the normal group. MSCT and MRI scanning were performed on patients in both groups to analyze their diagnostic value for stages $\mathrm{A} / \mathrm{B}$ and $\mathrm{C} / \mathrm{D}$ of prostate cancer. Based on the apparent diffusion coefficient (ADC) value generated by the diffusion-weighted imaging (DWI) in MRI, there was a significant difference in the ADC value between different stages of prostate cancer $(\mathrm{P}<0.05)$; the pathological stage was negatively correlated with the ADC value $(\mathrm{r}=-0.7629, \mathrm{P}<0.05)$, and the higher the stage was, the lower the ADC value was. The sensitivity was significantly higher in the MRI group than that in the MSCT group ( 92.0 vs. $79.5 \%, \mathrm{P}<0.05)$, and the specificity was significantly higher in the MRI group than that in the MSCT group (90.0 vs. $70.0 \%, \mathrm{P}<0.05)$. In the diagnosis of stage $\mathrm{A}$ and $\mathrm{B}$ of prostate cancer, the diagnostic coincidence rate was $86.7 \%$ in the MRI group, and $57.8 \%$ in the MSCT group $(\mathrm{P}<0.05)$; the misdiagnosis rate and missed diagnosis rate were significantly lower in the MRI group than those in the MSCT group $(\mathrm{P}<0.05)$. The accuracy of MRI is higher than that of MSCT in the diagnosis of early prostate cancer. Both MRI and MSCT can accurately detect stages C and D of prostate cancer, but the ADC value in MRI has great clinical
\end{abstract}

Correspondence to: Dr Cuijuan Hao, Department of Medical Image, The Affiliated Yantai Yuhuangding Hospital of Qingdao University, 20 Yuhuangding East Road, Yantai, Shandong 264000, P.R. China

E-mail: ey36ga@163.com

*Contributed equally

Key words: multi-slice spiral CT, magnetic resonance imaging, pathological stage, prostate cancer, diagnostic value significance for judging the risk of the tumor. Therefore, MRI is more valuable than MSCT in the diagnosis of patients with different pathological stages of prostate cancer.

\section{Introduction}

Prostate cancer, one of the leading causes of death in the male population in Europe and the United States, ranks first among male urogenital diseases (1). The incidence of prostate cancer is increasing with the continuous improvement of people's material living standards $(2,3)$. The 5-year survival rate of patients with metastatic prostate cancer is $36 \%$ to $54 \%$ (4). This may be due to the difficulty in the treatment of advanced prostate cancer. Radical resection for treating patients with early prostate cancer greatly improves their quality of life and survival quality (5). At present, the cause of prostate cancer remains unclear. Its treatment is still in the experimental stage, and the conditions for clinical large-scale application are not yet available. Therefore, it is especially important to select the best clinically diagnostic method for the early diagnosis of prostate cancer (6).

Various advanced science and technology have been widely used in the diagnosis of clinical diseases due to their continuous advancement and development. Radiological examinations such as magnetic resonance imaging (MRI) and computed tomography (CT) have good diagnostic value for the diagnosis and stage of prostate cancer. This is particularly true for the multi-parameter MRI with MRI combined with spectrum analysis and dynamic diffusion-weighted imaging (DWI), which is increasingly recognized in clinical practice and has a great value in the early diagnosis and clinical stage of prostate cancer (7). There is literature showing that the diagnostic sensitivity of CT is lower than that of MRI in early prostate cancer (8). Multi-slice spiral CT (MSCT) scanning is considered to be more sensitive and specific than traditional CT scanning in detecting lymph node metastasis of carcinoma of esophagus (9). However, there are relatively few comparative studies on the use of MSCT and MRI in the diagnosis of patients with different pathological stages of prostate cancer. In this investigation, the accuracy of MSCT and MRI was compared in the detection of stages $\mathrm{A} / \mathrm{B}$ and $\mathrm{C} / \mathrm{D}$ of prostate cancer, and their sensitivity and specificity were also compared, in order to explore the diagnostic value for the different pathological stages of prostate cancer. 


\section{Patients and methods}

General information. A total of 112 patients with prostate cancer who underwent surgical pathology in The Affiliated Yantai Yuhuangding Hospital of Qingdao University (Yantai, China) from February 2014 to January 2016 were enrolled as the prostate cancer group. They were aged $62.34 \pm 9.65$ years, with a course of disease of 6 months to 4 years and an average course of disease of $2.0 \pm 1.2$ years. The serum prostatespecific antigen detection value (PSA) of the patients was $25.23 \pm 9.9 \mathrm{ng} / \mathrm{ml}$, and there were 89 patients with an increase in single serum PSA (Table I). Another 100 patients who received physical health examinations in The Affiliated Yantai Yuhuangding Hospital of Qingdao University during the same period were collected as the normal group, aged $62.65 \pm 9.57$ years.

Inclusion criteria: Patients who did not receive anti-tumor treatments before the examination; patients with clinical symptoms that were mainly frequent micturition, urgency of urination, dysuria and frequent nocturia, and some patients with hematuria; males aged over 50 years.

Exclusion criteria: Patients with other severe tumor diseases; patients who did not actively cooperate; patients who did not undergo routine examination before operation; those previously suffering from mental disorder and with a family history of mental illness; those with incomplete clinical data.

The study was approved by the Ethics Committee of The Affiliated Yantai Yuhuangding Hospital of Qingdao University and the experimental content of subjects was described in detail. Subjects agreed to and signed a completed informed consent form.

\section{Detection methods}

MSCT examination. In this study, the Toshiba Activion 16-slice spiral CT instrument was used for MSCT examination. Each patient was placed in the supine position and scanned, and the scan range included the prostate, the seminal vesicle and the bladder. Perfusion scanning was performed centering on the largest central level of the lesion or the center level of the prostate. Scanning parameters were as follows: $320 \mathrm{~mA}$ in current flow, $120 \mathrm{kV}$ in voltage, 0.9376 in pitch, with $16 \times 0.5 \mathrm{~mm}$ thin layer detector. The enhanced scanning was performed with a non-ionic contrast media. The dosage of the contrast agent (Bayer Schering Pharmaceutical Co., Ltd.; guoyaozhunzi: J20050047) was adjusted to $1.5-2.0 \mathrm{ml} / \mathrm{kg}$ based on the actual situation of the patients, and was injected from the median vein of the elbow at a flow rate of $3.5-4 \mathrm{~m} / \mathrm{sec}$. Then, dynamic scanning was performed $5 \mathrm{sec}$ later, with a scanning time of $0.5 \mathrm{sec} / 16$ layers. The data was reconstructed, with $0.8 \mathrm{~mm}$ in reconstruction interval and $1 \mathrm{~mm}$ in layer thickness, and then transmitted to the processing server. The imaging was reconstructed with multi-planar reconstruction (MPR), maximum density projection (MIP) and volume rendering technology (VR). The attending radiologists (at least two) analyzed the data together and made a conclusion.

MRI examination. The HDXT 1.5T superconducting MRI imager from GE was used as an MRI diagnostic instrument. Before the scanning, the patients were told to drink water in order to maintain the filling of the bladder. During the operation, patients were placed in the supine position and maintained uniform and gentle breathing. Array coils were performed through the body phase control of standard 6 units. The scanning sequence was conventional coronal, sagittal, cross-sectional T1WI, T2WI and DWI images. Specific parameters were as follows: T1WI was $(\mathrm{TR} / \mathrm{TE}=250 / 4.92 \mathrm{msec}$, matrix $320 * 256)$; T2WI was $(\mathrm{TR} / \mathrm{TE}=6,000 / 100 \mathrm{msec}$, matrix $320 * 320$ ); the number of excitations was 2 to 4 times, FOV was $240 * 240 \mathrm{~mm}$, the layer thickness was $5 \mathrm{~mm}$, and the interlayer spacing was $5 \mathrm{~mm}$. Chemical displacement Selection Saturation method was used for lipid inhibition. DWI was $(\mathrm{TR} / \mathrm{TE}=8,200 / 100 \mathrm{msec}$, matrix $128 * 128$, the layer thickness was $5 \mathrm{~mm}$; the interlayer spacing was $5 \mathrm{~mm}$, and the $b$-value was $1,000 \mathrm{sec} / \mathrm{mm}^{2}$ ).

Criteria for clinical stage. The Whitmore-Jewett method (10) was used for staging prostate cancer that was divided into stage A, B, C and D. In stage A, the tumor was concealed in the prostate and difficult to be detected through the rectal mouth. In stage B, the tumor could be detected in the rectum, and there was a tumor in the capsule of the prostate. In stage $\mathrm{C}$, the tumor had exceeded the capsule of the prostate, but there was no metastasis. In stage D, the tumor had a distant metastasis.

Outcome measures. The apparent diffusion coefficient (ADC) plot was automatically generated after the DWI scanning. The ADC value of the lesion was measured by manually placing the region of interest (ROI) on the ADC plot. Calcifications, blood vessels or bleeding were avoided, and the corresponding ADC values were recorded. The measurement was repeated twice to calculate the average value.

Clinical outcome measures: The sensitivity and specificity of MSCT and MRI for the diagnosis of prostate cancer were calculated based on the results of postoperative puncture pathological biopsy. The formula was as follows: the sensitivity $=[$ true positive $/($ true positive + false negative) $\times 100 \%]$, and the specificity $=[$ true negative/(true negative + false positive) $\mathrm{x} 100 \%$. The diagnostic coincidence rates, misdiagnosis rates and missed diagnosis rates of MSCT and MRI for prostate cancer staging were compared.

Statistical analysis. SPSS 17.0 statistical software (SPSS Inc., Chicago, IL, USA) was used for the statistical analysis of the experimental data. Enumeration data were expressed as $\mathrm{n}(\%)$, and Chi-square test was used for comparison between the two groups. Measurement data were expressed as (mean $\pm \mathrm{SD}$ ), one-way analysis of variance and LSD post hoc test were used for the comparison of mean between multiple groups, and paired t-test was used between the two groups. Spearman correlation coefficient was used for analyzing the pathological stage and the ADC value. $\mathrm{P}<0.05$, indicates the difference is statistically significant.

\section{Results}

Comparison of clinical baseline data between the two groups. The clinical baseline data of age, height, body mass index, smoking and drinking, presence or absence of diabetes, white blood cells (WBC), hemoglobin (HB), red blood cell (RBC) count and platelet (PLT) count were collected from patients 
Table I. Patient general information $[\mathrm{n}(\%)] /($ mean $\pm \mathrm{SD})$.

\begin{tabular}{lc}
\hline Variables & $\begin{array}{c}\text { Patients with } \\
\text { prostate cancer } \\
(\mathrm{n}=112)\end{array}$ \\
\hline Age (years) & $62.34 \pm 9.65$ \\
Ethnicity & $69(61.6)$ \\
Han & $43(38.4)$ \\
Ethnic minority & 6 months-4 years \\
Course of disease & $2.0 \pm 1.2$ \\
Average course of disease (years) & $43.13 \pm 19.89$ \\
Prostate volume (ml) & $25.23 \pm 9.9$ \\
Serum PSA value (ng/ml) & $89(79.5)$ \\
Increased single serum PSA (patients) & $19(17.0)$ \\
Positive rectal touch (patients) & $15(13.4)$ \\
Infringement of seminal vesicle (patients) & $11(9.8)$ \\
Infringement of the bladder (patients) & $4(3.6)$ \\
Iliac bone metastasis (patients) & $8(7.1)$ \\
Pulmonary metastasis (patients) & $11(9.8)$ \\
Pelvic lymph node metastasis (patients) &
\end{tabular}

in the prostate cancer group and the normal group. There was no statistically significant difference in the clinical baseline data of patients between the two groups $(\mathrm{P}>0.05)$, which are comparable (Table II).

Comparison of $A D C$ values between different stages of prostate cancer. Based on the ADC values generated by DWI imaging in MRI (Table III), the ADC value of stage A was significantly higher than that of stage $\mathrm{B}, \mathrm{C}$ and $\mathrm{D}(\mathrm{P}<0.05)$, that of stage $\mathrm{B}$ was significantly higher than that of stage $C$ and $D(P<0.05)$, and that of stage $C$ was significantly higher than of stage $D$ $(\mathrm{P}<0.05)$. The pathological stage was negatively correlated with the ADC value $(r=-0.7629, \mathrm{P}<0.05)$, indicating that the ADC value correlates well with the tumor stage, and the higher the stage is, the lower the ADC value is (Fig. 1).

Diagnostic results of prostate cancer by MSCT, MR and pathology. Altogether 112 patients with prostate cancer were detected by puncture pathological biopsy. In total 113 positives were detected by MRI, of which 103 positives were true positives and 10 positives were false positives. Altogether 119 positives were detected by MSCT, of which 89 positives were true positives and 30 positives were false positives (Table IV). As can be seen from Table $\mathrm{V}$, the diagnostic sensitivity of MRI was significantly higher than that of MSCT (92.0 vs. $79.5 \%, \mathrm{P}<0.05)$, and the diagnostic specificity of MRI was significantly higher than that of MSCT $(90.0$ vs. $70.0 \%, \mathrm{P}<0.05)$, with statistically significant differences.

Comparison of the diagnostic accuracy of stage A and B of prostate cancer in patients. The operation was performed on 112 patients with prostate cancer. A total of 22 patients with stage $\mathrm{A}$ of prostate cancer and 23 patients with stage $\mathrm{B}$ of

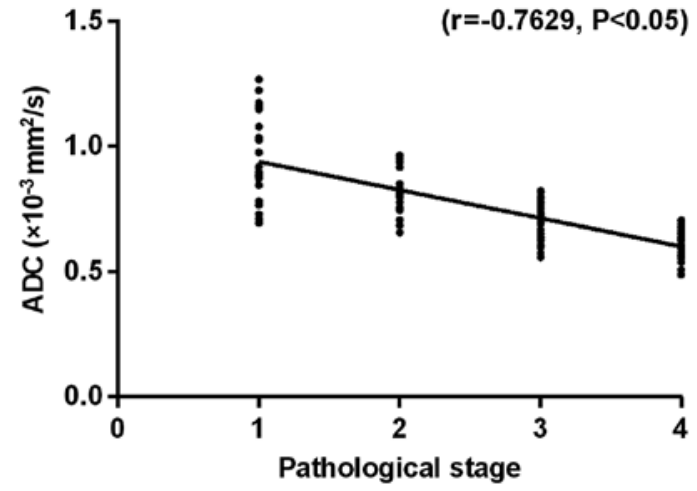

Figure 1. Comparison of ADC values between different stages of prostate cancer. ADC, apparent diffusion coefficient.

prostate cancer were diagnosed by pathological biopsy. A total of 26 patients with stages A and B of prostate cancer were diagnosed by MSCT, with a misdiagnosis rate of $17.8 \%$ and a missed diagnosis rate of $24.4 \%$. A total of 39 patients with stages A and B of prostate cancer were diagnosed by MRI, with a misdiagnosis rate of $4.4 \%$ and a missed diagnosis rate of $8.9 \%$. There was a significant difference in the diagnostic coincidence rate between MSCT and MRI $(\mathrm{P}<0.05)$. The misdiagnosis rate and missed diagnosis rate of MRI were significantly lower than those of MSCT $(\mathrm{P}<0.05)$ (Table VI).

Comparison of the diagnostic accuracy of stage $C$ and $D$ of prostate cancer in patients. The operation was performed on 112 patients with prostate cancer. A total of 38 patients stage $C$ of prostate cancer and 29 patients with stage D of prostate cancer were diagnosed by pathological biopsy. A total of 63 patients with stage $\mathrm{C}$ and $\mathrm{D}$ of prostate cancer were diagnosed by MSCT, with a misdiagnosis rate of $6.0 \%$. There was no missed diagnosis in MSCT and MRI. A total of 64 patients with stage $\mathrm{C}$ and $\mathrm{D}$ of prostate cancer were diagnosed by MRI, with a misdiagnosis rate of $4.5 \%$. There was no statistically significant difference in the diagnostic coincidence rate and misdiagnosis rate between the two groups $(\mathrm{P}>0.05)$ (Table VII).

\section{Discussion}

Prostate cancer is a male malignant tumor and its incidence is the highest among all male malignant tumors (11). It has been reported that its incidence in male tumors is $9.7 \%(12,13)$. The cause of prostate cancer currently remains unclear (14). Although China has a low incidence of prostate cancer, the incidence has shown a certain upward trend in recent years (3). Early detection, diagnosis and treatment can significantly improve the prognosis of prostate cancer (15).

At present, PSA is a commonly used method for detecting prostate cancer, but some studies have found that it can only be used as a preliminary screening method, and the detection results often cause unnecessary iatrogenic injuries (16). The key to the treatment and prognosis of prostate cancer is early diagnosis and stage (17). Imaging plays an important role. The spiral CT has improved the sharpness of the image, but there are sometimes artifacts during the $\mathrm{CT}$ examination, which interferes with the diagnosis (18). As a commonly used imaging method, MRI images in multiple directions. It has a high 
Table II. Comparison of clinical baseline data between the two groups.

\begin{tabular}{lcccc}
\hline & $\begin{array}{c}\text { Prostate cancer } \\
\text { group }(\mathrm{n}=112)\end{array}$ & $\begin{array}{c}\text { Normal group } \\
(\mathrm{n}=100)\end{array}$ & $\chi^{2} / \mathrm{t}$ & P-value \\
\hline Age $($ years $)$ & $62.34 \pm 9.65$ & $62.65 \pm 9.57$ & 0.234 & 0.815 \\
Height $(\mathrm{cm})$ & $167.23 \pm 7.87$ & $167.36 \pm 6.98$ & 0.127 & 0.899 \\
Body mass index $\left(\mathrm{kg} / \mathrm{m}^{2}\right)$ & $25.37 \pm 2.87$ & $25.76 \pm 2.65$ & 1.024 & 0.307 \\
Smoking & & & 0.017 & 0.897 \\
Yes & $82(73.2)$ & $74(74.0)$ & & 0.297 \\
No & $30(26.8)$ & $26(26.0)$ & & \\
Drinking & & & & \\
Yes & $76(67.9)$ & $61(61.0)$ & & 0.087 \\
No & $36(32.1)$ & $39(39.0)$ & & \\
History of diabetes mellitus & $48(42.9)$ & $39(39.0)$ & & \\
Yes & $64(57.1)$ & $61(61.0)$ & & 0.325 \\
No & $6.19 \pm 3.24$ & $6.23 \pm 3.35$ & 0.088 & 0.427 \\
WBC $\left(\mathrm{x} 10^{9} / \mathrm{l}\right)$ & $12.24 \pm 2.09$ & $12.47 \pm 2.11$ & 0.796 & 0.681 \\
HB $(\mathrm{gm} / \mathrm{dl})$ & $161.17 \pm 20.98$ & $159.98 \pm 21.09$ & 0.411 & 0.716 \\
PLT $\left(\mathrm{x} 10^{9} / \mathrm{l}\right)$ & $4.54 \pm 0.59$ & $4.57 \pm 0.61$ & 0.363 & \\
RBC $\left(10^{12} / \mathrm{l}\right)$ & & & & \\
\hline
\end{tabular}

WBC, white blood cells; HB, hemoglobin; PLT, count and platelet; RBC, red blood cell.

Table III. Comparison of ADC values between different stages of prostate cancer.

\begin{tabular}{lcc}
\hline Stage & Number of cases & $\begin{array}{c}\text { ADC value } \\
\left(\times 10^{-3} \mathrm{~mm}^{2} / \mathrm{sec}\right)\end{array}$ \\
\hline A & 22 & $0.92 \pm 0.18$ \\
B & 23 & $0.83 \pm 0.11^{\mathrm{a}}$ \\
$\mathrm{C}$ & 38 & $0.71 \pm 0.08^{\mathrm{a}, \mathrm{b}}$ \\
$\mathrm{D}$ & 29 & $0.61 \pm 0.05^{\mathrm{a}-\mathrm{c}}$ \\
F-value & & 40.56 \\
P-value & & $<0.0001$ \\
\hline
\end{tabular}

Compared with stage A, ${ }^{\mathrm{P}} \mathrm{P}<0.05$; Compared with stage $\mathrm{B},{ }^{\mathrm{b}} \mathrm{P}<0.05$; Compared with stage $\mathrm{C},{ }^{\mathrm{c}} \mathrm{P}<0.05$. ADC, apparent diffusion coefficient.

diagnostic value for finding the primary lesion of prostate cancer and determining the lesion size, local involvement (whether the tumor had broken through the capsule and whether it involved the seminal vesicle) range, and pelvic lymph node metastasis. It also shows lesions of bone metastasis (19). Being a new MRI functional imaging technology, DWI was developed in the early and middle 1990s. It is the only non-invasive method that reflects the phenomenon of the diffusion of the living tissue, and characterized by its sensitivity to molecular motion (20).

There is usually no obvious symptom in the early stage of prostate cancer. Once found, prostate cancer is in the advanced stage, so its clinical treatment effect is unsatisfactory (6). Prostate cancer is specifically manifested on MRI, CT and other examinations (21). Therefore, the comparison of the differences
Table IV. Diagnostic results of prostate cancer by MSCT, MR and pathology (patients).

\begin{tabular}{lccr}
\hline $\begin{array}{l}\text { MSCT/MRI } \\
\text { results }\end{array}$ & $\begin{array}{c}\text { Pathologic } \\
\text { findings (+) }\end{array}$ & $\begin{array}{c}\text { Pathologic } \\
\text { findings (-) }\end{array}$ & Total \\
\hline MRI $^{+}$ & 103 & 10 & 113 \\
MRI $^{-}$ & 9 & 90 & 99 \\
Total & 112 & 100 & 212 \\
MSCT $^{+}$ & 89 & 30 & 119 \\
MSCT $^{-}$ & 23 & 70 & 93 \\
Total & 112 & 100 & 212 \\
& & &
\end{tabular}

MRI, magnetic resonance imaging; MSCT, multi-slice spiral CT.

Table V. Comparison of sensitivity and specificity between the two groups $/ \%$.

\begin{tabular}{lcc}
\hline & Sensitivity & Specificity \\
\hline MRI & 92.0 & 90.0 \\
MSCT & 79.5 & 70.0 \\
$\chi^{2}$ & 6.66 & 12.50 \\
P-value & 0.01 & $<0.001$
\end{tabular}

MRI, magnetic resonance imaging; MSCT, multi-slice spiral CT.

between MSCT and MRI in the diagnosis of different stages of prostate cancer provides a certain reference value for clinical 
Table VI. Comparison of the diagnostic accuracy of stage A and B of prostate cancer in patients [n(\%)].

\begin{tabular}{lcccc}
\hline $\begin{array}{l}\text { Diagnostic } \\
\text { methods }\end{array}$ & $\begin{array}{c}\text { Number of } \\
\text { pathological findings }\end{array}$ & $\begin{array}{c}\text { Diagnostic } \\
\text { coincidence rate }\end{array}$ & $\begin{array}{c}\text { Missed diagnosis } \\
\text { rate }\end{array}$ & $\begin{array}{c}\text { Misdiagnosis } \\
\text { rate }\end{array}$ \\
\hline MSCT & 45 & $26(57.8)$ & $11(24.4)$ & $8(17.8)$ \\
MRI & 45 & $39(86.7)$ & $4(8.9)$ & $2(4.4)$ \\
$\chi^{2}$ & & 9.36 & 3.92 & 4.05 \\
P-value & & 0.002 & 0.048 & 0.044 \\
\hline
\end{tabular}

MRI, magnetic resonance imaging; MSCT, multi-slice spiral CT.

Table VII. Comparison of the diagnostic accuracy of stage C and D of prostate cancer in patients [n(\%)].

\begin{tabular}{lccc}
\hline $\begin{array}{l}\text { Diagnostic } \\
\text { methods }\end{array}$ & $\begin{array}{c}\text { Number of } \\
\text { pathological findings }\end{array}$ & $\begin{array}{c}\text { Diagnostic } \\
\text { coincidence rate }\end{array}$ & $\begin{array}{c}\text { Misdiagnosis } \\
\text { rate }\end{array}$ \\
\hline MSCT & 67 & $63(94.0)$ & $4(6.0)$ \\
MRI & 67 & $64(95.5)$ & $3(4.5)$ \\
$\chi^{2}$ & & 0.15 & 0.15 \\
P-value & & 0.698 & 0.698 \\
\hline
\end{tabular}

MRI, magnetic resonance imaging; MSCT, multi-slice spiral CT.

research. In this investigation, the basic information of patients with prostate cancer was compared with that of healthy controls in the normal control group, with no significant difference. The ADC value reflects the degree of diffusion of water molecules in the tissue, and the ADC value increases as the tissue signal with fast diffusion decays (22). This study found a negative correlation between the pathological stage and the ADC value. The higher the stage was, the lower the ADC value was. In the study by Anwar et al (23), the ADC value of prostate cancer was $0.57 \pm 0.08-0.94 \pm 0.25 \times 10^{3} \mathrm{~mm}^{2} / \mathrm{sec}$, poorly differentiated prostate cancer had a low ADC value, and well differentiated prostate cancer had a high ADC value. These findings indicate that the more severe the tumor is, the lower the ADC value is. The data of our study are similar to the results of that study and furthermore found that the number of true positives detected by MRI was significantly higher than that detected by MSCT, and the sensitivity and specificity were significantly higher in the MRI group than those in the MSCT group, with statistically significant differences $(\mathrm{P}<0.05)$. There is literature showing that the resolution of dynamic enhanced MRI in scanning soft tissue is higher than that of CT (8). In the diagnosis of stage A and B prostate cancer, the diagnostic coincidence rate was $86.7 \%$ in the MRI group, and $57.8 \%$ in the MSCT group, with a significant difference between the two groups $(\mathrm{P}<0.05)$. The misdiagnosis rate and missed diagnosis rate was significantly lower in the MRI group than those in the MSCT group $(\mathrm{P}<0.05)$. This may be because there is an increase in prostate volume in stage $A$ and $\mathrm{B}$ of prostate cancer, but no significant change in density. Besides, the blurring of the edge affects CT diagnosis (23). As a result, the accuracy is low. Therefore, MRI has an advantage in the diagnosis of early prostate cancer, with a low error rate. In the diagnosis of stage $\mathrm{C}$ and $\mathrm{D}$ of prostate cancer, there were no statistically significant differences in the diagnostic coincidence rate and misdiagnosis rate between the two groups $(\mathrm{P}>0.05)$. Both MRI and MSCT can accurately detect stage $\mathrm{C}$ and $\mathrm{D}$ of prostate cancer, considering that it is clearly related to the fact that the cancer tissue has penetrated the capsule, and the morphological changes of the prostate. MRI judges whether the capsule is attacked by cancer cells (24). Therefore, MRI is important for the diagnosis of different clinical stages, and shows clearly bone metastasis and the lesion invasion of pelvic lymph nodes (25), which has a great application value.

In the study, the sensitivity, specificity and accuracy of MSCT and MRI were compared. However, there is no unified diagnosis of patients with prostate cancer in clinical practice. Therefore, the research in this direction should be increased in the future, and the prognosis of patients should be discussed in depth, to improve the diagnosis rate and reduce the deterioration of the disease.

The accuracy of MRI is higher than that of MSCT in the diagnosis of patients with stage A and B of prostate cancer, but that of MSCT and MRI is similar in the diagnosis of patients with stage $C$ and $D$ of prostate cancer. The sensitivity and specificity of MRI diagnosis are higher than those of MSCT, and the ADC value in MRI has great clinical significance for judging the risk of a tumor. Therefore, MRI is more valuable than MSCT in the diagnosis of patients with different pathological stages of prostate cancer.

\section{Acknowledgements}

Not applicable.

\section{Funding}

No funding was received. 


\section{Availability of data and materials}

The datasets used and/or analyzed during the present study are available from the corresponding author on reasonable request.

\section{Authors' contributions}

YS and JL conceived and designed the study. $\mathrm{CH}$ collected the patients' data. ZZ and YS analyzed and interpreted the patient data regarding the different pathological stages of prostate cancer. YS, JL, ZZ and YS were the major contributors in writing the manuscript. $\mathrm{CH}$ reviewed the manuscript. All authors read and approved the final manuscript.

\section{Ethics approval and consent to participate}

The study was approved by the Ethics Committee of The Affiliated Yantai Yuhuangding Hospital of Qingdao University (Yantai, China). Patients who participated in this research had complete clinical data. Signed informed consents were obtained from the patients or the guardians.

\section{Patient consent for publication}

Not applicable.

\section{Competing interests}

The authors declare that they have no competing interests.

\section{References}

1. Osimani M, Bellini D, Di Cristofano C, Palleschi G, Petrozza V, Carbone A and Laghi A: Perfusion MDCT of prostate cancer: Correlation of perfusion CT parameters and immunohistochemical markers of angiogenesis. AJR Am J Roentgenol 199: 1042-1048, 2012.

2. Resnick MJ, Koyama T, Fan KH, Albertsen PC, Goodman M, Hamilton AS, Hoffman RM, Potosky AL, Stanford JL, Stroup AM, et al: Long-term functional outcomes after treatment for localized prostate cancer. N Engl J Med 368: 436-445, 2013.

3. Chen W, Zheng R, Baade PD, Zhang S, Zeng H, Bray F, Jemal A, Yu XQ and He J: Cancer statistics in China, 2015. CA Cancer J Clin 66: 115-132, 2016.

4. Maresca KP, Hillier SM, Femia FJ, Keith D, Barone C, Joyal JL, Zimmerman CN, Kozikowski AP, Barrett JA, Eckelman WC, et al: A series of halogenated heterodimeric inhibitors of prostate specific membrane antigen (PSMA) as radiolabeled probes for targeting prostate cancer. $\mathbf{J}$ Med Chem 52: 347-357, 2009.

5. Mottet N, Bellmunt J, Bolla M, Briers E, Cumberbatch MG, De Santis M, Fossati N, Gross T, Henry AM, Joniau S, et al: EAU-ESTRO-SIOG Guidelines on Prostate Cancer. Part 1: Screening, Diagnosis, and Local Treatment with Curative Intent. Eur Urol 71: 618-629, 2017.

6. Shen G, Deng H, Hu S and Jia Z: Comparison of choline-PET/CT, MRI, SPECT, and bone scintigraphy in the diagnosis of bone metastases in patients with prostate cancer: A meta-analysis. Skeletal Radiol 43: 1503-1513, 2014.

7. Stephenson SK, Chang EK and Marks LS: Screening and detection advances in magnetic resonance image-guided prostate biopsy. Urol Clin North Am 41: 315-326, 2014.

8. Afshar-Oromieh A, Haberkorn U, Schlemmer HP, Fenchel M, Eder M, Eisenhut M, Hadaschik BA, Kopp-Schneider A and Röthke M: Comparison of PET/CT and PET/MRI hybrid systems using a $68 \mathrm{Ga}$-labelled PSMA ligand for the diagnosis of recurrent prostate cancer: Initial experience. Eur J Nucl Med Mol Imaging 41: 887-897, 2014.
9. Tan R, Yao SZ, Huang ZQ, Li J, Li X, Tan HH and Liu QW: Combination of FDG PET/CT and contrast-enhanced MSCT in detecting lymph node metastasis of esophageal cancer. Asian Pac J Cancer Prev 15: 7719-7724, 2014.

10. Tobisu K: Clinical and pathological staging of prostate cancer. Nihon Rinsho 63: 225-230, 2005 (In Japanese).

11. Siegel RL, Miller KD and Jemal A: Cancer Statistics, 2017. CA Cancer J Clin 67: 7-30, 2017.

12. Liu Y, Gao X, Deeb D, Zhang Y, Shaw J, Valeriote FA and Gautam SC: Mycotoxin verrucarin A inhibits proliferation and induces apoptosis in prostate cancer cells by inhibiting prosurvival Akt/NF- $\mathrm{kB} / \mathrm{mTOR}$ signaling. J Exp Ther Oncol 11: 251-260, 2016.

13. Shen YC, Kang $\mathrm{CH}$ and Chiang PH: Efficacy of switching therapy of luteinizing hormone-releasing hormone analogue for advanced prostate cancer. Kaohsiung J Med Sci 32: 567-571, 2016.

14. Tsai YC, Chen WY, Siu MK, Tsai HY, Yin JJ, Huang J and Liu YN: Epidermal growth factor receptor signaling promotes metastatic prostate cancer through microRNA-96-mediated downregulation of the tumor suppressor ETV6. Cancer Lett 384: 1-8, 2017.

15. Turkbey B, Mani H, Shah V, Rastinehad AR, Bernardo M, Pohida T, Pang Y, Daar D, Benjamin C, McKinney YL, et al: Multiparametric 3T prostate magnetic resonance imaging to detect cancer: Histopathological correlation using prostatectomy specimens processed in customized magnetic resonance imaging based molds. J Urol 186: 1818-1824, 2011.

16. Bell N, Connor Gorber S, Shane A, Joffres M, Singh H, Dickinson J, Shaw E, Dunfield L and Tonelli M; Canadian Task Force on Preventive Health Care: Recommendations on screening for prostate cancer with the prostate-specific antigen test. CMAJ 186: 1225-1234, 2014.

17. Jemal A, Siegel R, Ward E, Hao Y, Xu J and Thun MJ: Cancer statistics, 2009. CA Cancer J Clin 59: 225-249, 2009.

18. Zhang X, Quan X, Lu S, Huang F, Yang J, Chan Q and Lin T: The clinical value of dynamic contrast-enhanced magnetic resonance imaging at 3.0T to detect prostate cancer. J Int Med Res 42: 1077-1084, 2014.

19. Frauscher F, Halpern EJ and Klauser A: Use of MRI to detect lymph-node metastases in prostate cancer. N Engl J Med 349: 1185-1186, 2003

20. Eiber M, Holzapfel K, Ganter C, Epple K, Metz S, Geinitz H, Kübler H, Gaa J, Rummeny EJ and Beer AJ: Whole-body MRI including diffusion-weighted imaging (DWI) for patients with recurring prostate cancer: Technical feasibility and assessment of lesion conspicuity in DWI. J Magn Reson Imaging 33: 1160-1170, 2011.

21. Volkin D, Turkbey B, Hoang AN, Rais-Bahrami S, Yerram N, Walton-Diaz A, Nix JW, Wood BJ, Choyke PL and Pinto PA: Multiparametric magnetic resonance imaging (MRI) and subsequent MRI/ultrasonography fusion-guided biopsy increase the detection of anteriorly located prostate cancers. BJU Int 114: E43-E49, 2014

22. Tanimoto A, Nakashima J, Kohno H, Shinmoto $H$ and Kuribayashi S: Prostate cancer screening: The clinical value of diffusion-weighted imaging and dynamic MR imaging in combination with T2-weighted imaging. J Magn Reson Imaging 25: 146-152, 2007.

23. Anwar SS, Anwar Khan Z, Shoaib Hamid R, Haroon F, Sayani R, Beg $M$ and Khattak YJ: Assessment of apparent diffusion coefficient values as predictor of aggressiveness in peripheral zone prostate cancer: Comparison with Gleason score. ISRN Radiol 2014: 263417, 2014.

24. Eschmann SM, Pfannenberg AC, Rieger A, Aschoff P, Müller M, Paulsen F, Anastasiadis A, Claussen CD, Bares R and Schlemmer HP: Comparison of 11C-choline-PET/CT and whole body-MRI for staging of prostate cancer. Nuklearmedizin 46: 161-168; quiz N47-N48, 2007.

25. Panebianco V, Sciarra A, Lisi D, Galati F, Buonocore V, Catalano C, Gentile V, Laghi A and Passariello R: Prostate cancer: 1HMRS-DCEMR at 3T versus [(18)F]choline PET/CT in the detection of local prostate cancer recurrence in men with biochemical progression after radical retropubic prostatectomy (RRP). Eur J Radiol 81: 700-708, 2012.

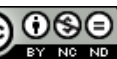

This work is licensed under a Creative Commons Attribution-NonCommercial-NoDerivatives 4.0 International (CC BY-NC-ND 4.0) License. 